\title{
Arabidopsis' hidden potential
}

The ability of heat-shock protein 90 (HSP90) to mask genetic polymorphisms and reveal them only in times of environmental instability has attracted attention because of its potential to mediate rapid evolutionary change. But how far reaching is the influence of HSP90 - that is, how many polymorphisms does it conceal? Two papers now report that the buffering mechanism is felt across the genome of Arabidopsis thaliana, where HSP90-masked polymorphisms influence quantitative traits and developmental stability.

HSP90 is a molecular chaperone - because its function is to stabilize protein conformation, it might dampen the effect of mutations that influence the folding of a protein, and therefore its function. When

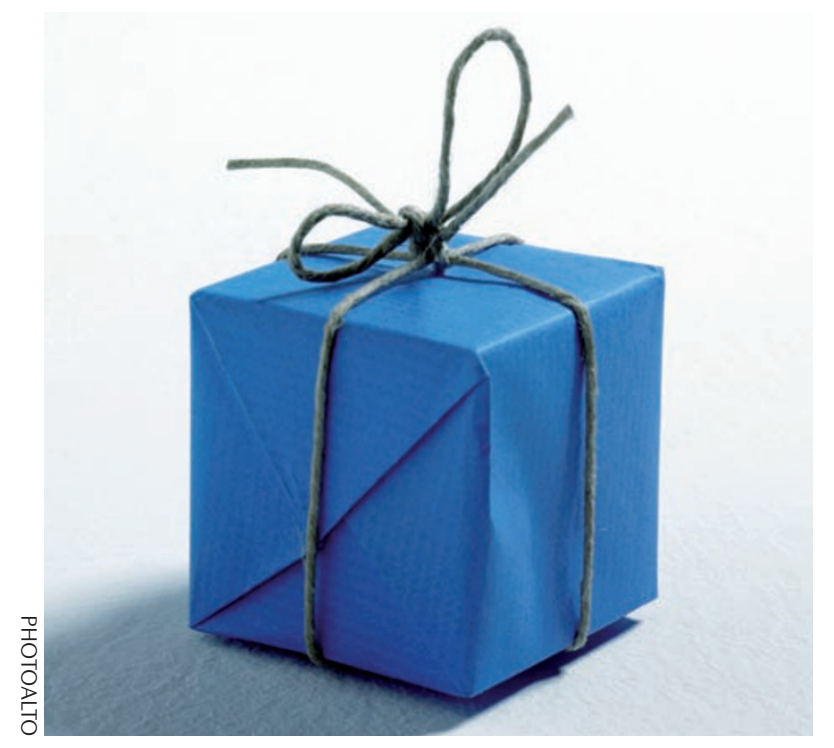

environmental circumstances change, or when HSP90 levels are experimentally reduced, these otherwise silent polymorphisms are exposed and can produce phenotypic variation. HSP90 has therefore been proposed as being a means of producing rapid evolutionary change. But any test of such a hypothesis needs hard data - that is, details of the polymorphisms themselves. This is what the authors sought to find by turning to A. thaliana, an organism that can be genetically manipulated and boasts many well-characterized, genetically diverse, inbred populations.

In the first paper, Sangster and colleagues aimed to map the effect of HSP90 on several life-history traits. Recombinant inbred lines (RILs) were generated between two A. thaliana strains in which the expression of HSP90 was constitutively reduced owing to an RNAi transgene. Using a genotyping array, high-density physical maps for these lines were created and numerous phenotypic traits were measured. Statistical association of phenotypes and genotypes yielded QTLs that were revealed only in the HSP90-reduced population. The authors found approximately 0.5 HSP90-buffered QTLs per chromosome per trait. One of these QTLs, for flowering time, was validated with near-isogenic lines that segregate only at the QTL region.

The second paper used a similar quantitative genetics approach to map HSP90-dependent polymorphisms and developmental stability of early seedling traits - the elongation of embryonic stems and roots in the dark. Embryonic stems normally elongate in the dark but growth is suppressed in the light; when HSP90 was inhibited, the natural variation among RILs in stem and root elongation changed markedly. The most plausible interpretation is that HSP90 normally suppresses genotypic variation for stem and root elongation in response to the environment. Indeed, the authors found multiple HSP90-dependent QTLs in three independent RIL populations. They also demonstrated that developmental stability - the degree to which isogenic individuals produce the same phenotype - has a genetic basis and can be affected by HSP90.

In the process of proving that HSP90-dependent alleles can be mapped genetically, these papers settle some debated issues surrounding the evolutionary function of HSP90 - the amount of variation available to HSP90 is larger, more varied, and less deleterious than previously thought. The effects of genetic variation outweigh those of developmental stability, thereby demonstrating that selection could act to increase the frequency of revealed polymorphisms in the population.

Tanita Casci

ORIGINAL RESEARCH PAPERS

Sangster, T. A. et al. HSP90-buffered genetic variation is common in Arabidopsis thaliana. Proc. Natl Acad. Sci. USA 19 Feb 2008 (doi:10.1073/ pnas.0712210105)|Sangster, T. A. et al. HSP90 affects the expression of genetic variation and developmental stability in quantitative traits. Proc. Natl Acad. Sci. USA 19 Feb 2008 (doi: 10.1073/pnas.0712200105) 